\title{
Can Macula and Optic Nerve Head Parameters Detect Glaucoma Progression in Eyes with Advanced Circumpapillary Retinal Nerve Fiber Layer Damage?
}

\author{
Fabio Lavinsky ${ }^{1}$, Mengfei Wu ${ }^{1,2}$, Joel S. Schuman ${ }^{1}$, Katie A. Lucy ${ }^{1}$, Mengling Liu², \\ Youngseok Song ${ }^{1}$, Julia Fallon ${ }^{1}$, Maria de Los Angeles Cadenaa ${ }^{1}$, Hiroshi Ishikawa ${ }^{1}$, and \\ Gadi Wollstein ${ }^{1}$ \\ ${ }^{1}$ NYU Langone Eye Center, New York University School of Medicine, New York, NY \\ ${ }^{2}$ Division of Biostatistics, Departments of Population Health and Environmental Medicine, NYU \\ School of Medicine, New York, NY
}

\begin{abstract}
Purpose-To evaluate the ability of OCT optic nerve head (ONH) and macular parameters to detect disease progression in eyes with advanced structural glaucomatous damage of the circumpapillary retinal nerve fiber layer (cRNFL).
\end{abstract}

Design-Longitudinal study.

Participants-Forty-four eyes from 37 subjects with advanced average cRNFL damage ( $\$ 60$ $\mu \mathrm{m})$ followed for an average period of 4.0 years.

\begin{abstract}
Methods-All subjects were examined with spectral-domain optical coherence tomography (SDOCT) and visual field (VF) during at least 4 visits.
\end{abstract}

Main Outcome Measurements-Visual field (VF) mean deviation (MD) and visual field index (VFI). OCT cRNFL (average, superior and inferior quadrants), ganglion cell/inner plexiform layer (GCIPL) (average, superior and inferior), rim area, cup volume, average cup-to-disc (C/D) ratio, vertical C/D ratio.

Results-At baseline, subjects had a median VF mean deviation (MD) of $-10.18 \mathrm{~dB}$ and mean cRNFL of $54.55 \pm 3.42 \mu \mathrm{m}$. The rate of change for MD and VFI were significant. No significant rate of change was noted for $\mathrm{cRNFL}$, while significant $(\mathrm{p}<0.001)$ rates were detected for GCIPL $(-0.57 \pm 0.05 \mu \mathrm{m} / \mathrm{yr})$ and $\mathrm{ONH}$ parameters such as rim area $\left(-0.010 \pm 0.001 \mathrm{~mm}^{2} / \mathrm{yr}\right)$.

Conclusions-Macula GCIPL and ONH parameters might be useful in tracking progression in subjects with advanced glaucoma.

\footnotetext{
Corresponding author: Joel S Schuman, MD, NYU Eye Center, NYU Langone Health, Department of Ophthalmology, New York University School of Medicine, 222 East $41^{\text {st }}$ Street, New York, NY 10017; joel.schuman@ nyu.edu.

Publisher's Disclaimer: This is a PDF file of an unedited manuscript that has been accepted for publication. As a service to our customers we are providing this early version of the manuscript. The manuscript will undergo copyediting, typesetting, and review of the resulting proof before it is published in its final citable form. Please note that during the production process errors may be discovered which could affect the content, and all legal disclaimers that apply to the journal pertain.

Conflict of Interest: Dr. Schuman receives royalties for intellectual property licensed by Massachusetts Institute of Technology and Massachusetts Eye and Ear Infirmary to Zeiss.
} 
Glaucoma is a multifactorial optic neuropathy leading to progressive damage to retinal ganglion cells and their axons in the retinal nerve fiber layer and thinning of the neuroretinal rim of the optic nerve head $(\mathrm{ONH})$. Damage to these structures leads to a characteristic cupping of the $\mathrm{ONH}$ and vision loss. ${ }^{1,2}$ Considering that glaucomatous damage is irreversible, sensitive methods for detecting progression are desirable in order to adjust treatment and prevent further damage. The introduction of ocular imaging technologies, such as optical coherence tomography (OCT) revolutionized glaucoma management, as it allows automated quantification of these structures and detection of small changes over time. 3-6 The evolution of OCT with faster scan speeds, improved resolution and reproducibility further improved the capability of glaucoma progression detection. Furthermore, automated segmentation of the macula, $\mathrm{ONH}$ and peripapillary regions enable multiple parameters to be used for monitoring the disease progression. . $^{7}$

Notwithstanding the improvements in OCT glaucoma longitudinal analysis tools, monitoring of progression in subjects with advanced glaucoma remains challenging. Many publications have demonstrated the ability of the circumpapillary retinal nerve fiber layer (cRNFL) to detect glaucoma progression. ${ }^{9}, 10$ However, in advanced glaucoma, the cRNFL reaches a minimum practical measurement ("floor effect") that restricts its usefulness for detecting disease progression beyond this point. ${ }^{11,12}$ Concurrently, there are also limitations in detecting progression with visual field tests when the threshold sensitivity is below $15 \mathrm{~dB}$ due to limited reproductibility. ${ }^{13,14}$ Taken together, detection of disease progression at advanced stages of the disease is clinically challenging.

Because central vision is often preserved until very late stages of glaucoma, we hypothesized that the macular inner retina and $\mathrm{ONH}$ parameters are a better location than the cRNFL for monitoring glaucoma progression in advanced disease. In the present study, we evaluated the ability of the ganglion cell inner plexiform layer (GCIPL), cRNFL and ONH parameters provided by commercially available spectral-domain (SD-) OCT software to detect glaucoma progression in subjects with structurally defined advanced disease.

\section{Methods}

Glaucoma subjects were enrolled from our ongoing prospective, longitudinal study designed to assess ocular structure over time. The institutional review boards and ethics committees at New York University and the University of Pittsburgh approved the study. The study followed the tenets of the Declaration of Helsinki and was conducted in compliance with the Health Insurance Portability and Accountability Act (HIPAA). Informed consent was obtained from all subjects.

\section{Subjects}

Subjects with advanced glaucoma were recruited to this study if they were 40 years old or older, had no history of diabetes or any systemic disease or medication usage that might affect the visual system and no major ocular trauma or ocular surgeries other than uncomplicated cataract or glaucoma surgeries performed prior to enrollment. Subjects had best corrected visual acuity of $20 / 60$ or better, spherical equivalent within $\pm 6 \mathrm{D}$, no media 
opacity precluding reliable VFs or OCT scanning and no intra-ocular co-morbidity other than glaucoma. All subjects had at least 4 visits, each of them at least 5 months apart, unless otherwise medically indicated. The maximum follow-up length allowed was 7 years. Subjects who underwent cataract or glaucoma surgery during the follow-up period were included either before or after the surgery as long as they had a sufficient number of visits.

Glaucoma diagnosis required at least two consecutive reliable VFs with glaucoma hemifield tests outside normal limits, or a cluster of three or more non-edge points in a location typical for glaucoma, with all points depressed on the pattern deviation plot at the $\mathrm{p}<5 \%$ level and at least one point depressed at the $\mathrm{p}<1 \%$ level. ${ }^{15}$ Advanced glaucoma was defined as eyes with baseline SD-OCT mean cRNFL thickness of $60 \mu \mathrm{m}$ or less.

\section{Study Protocol}

All subjects had their medical records reviewed and underwent a full ocular examination including refraction, best-corrected visual acuity (BCVA), biomicroscopy, Goldman applanation tonometry, gonioscopy and fundus examination. Visual field was tested with Swedish interactive thresholding algorithm (SITA standard; Humphrey Field Analyzer; Zeiss, Dublin, CA) 24-2 perimetry. Reliable VF were considered tests with less than 33\% fixation losses, false positive and false negative responses. Mean deviation (MD) and visual field index (VFI) were used for the analysis. All subjects were imaged with SD-OCT (Cirrus HD-OCT; Zeiss, Dublin CA) using the Macular Cube 200×200 and the Optic Disc Cube $200 \times 200$ scans. Scans were disqualified if they had a signal strength of less than 7 , decentration of the cRNFL sampling circle, segmentation errors or motion artifacts defined as a discontinuity of the blood vessels that exceeded the width of 1 major vessel diameter. Mean cRNFL (global and quadrants), macular GCIPL (global and sectorial), vertical cup/ disc (C/D) ratio and average C/D ratio, rim area and cup volume were used for the analysis.

Guided progression analysis (GPA) is a linear regression method provided by the OCT machine to determine disease progression. The proportion of eyes showing a significant rate of progression (also referred to as trend-based progression in this manuscript) for cRNFL and GCIPL was recorded. The GPA also reports change from baseline for these parameters that significantly exceeds expected population-derived change as progression (event-based progression). Eyes labeled by the software as "possible" or "likely" progression were considered to be progressing eyes.

\section{Statistical Analysis}

Rate of progression were calculated from longitudinal OCT and VF data using hierarchical linear models (HLMs) adjusting for baseline age, ethnicity, follow-up duration and signal strength at each visit and accounting for intra-subject correlation between eyes. To evaluate the influence of different lengths of follow-up on the rates of progression for OCT and VF parameters, a sensitivity analysis was also conducted where a non-linear quadratic term representing the follow-up length was included in the HLMs. A slope of progression with a $\mathrm{p}$ value $<0.05$ was considered statistically significant. $\mathrm{R}$ language and environment for statistical computing program (version 3.3.2) was used for statistical analysis. 


\section{Results}

Forty-four eyes from 37 subjects qualified for the study. The average age of the population at baseline was 67.0 years $(\mathrm{SD}=11.4)$. Twenty-one subjects were female $(57 \%)$ and the ethnicity profile was: 26 Caucasians (70\%), 10 African American (27\%) and 1 Asian (3\%). The mean follow-up duration was 4.0 years $(\mathrm{SD}=1.6)$ with $6.5(\mathrm{SD}=2.5)$ average total number of visits. Baseline VF and OCT parameters are summarized in Table 1.

The GPAs of the ONH showed two eyes with RNFL trend progression and 34 eyes with event progression. Only one of these eyes was labeled as progressing by both trend and event analysis. The average Cup-to-Disc analysis detected four progressing eyes (trend analysis). Fifteen eyes progressed by the GCIPL trend analysis, and 10 progressed by the event analysis, out of which nine eyes progressed by both trend and event analysis.

Age and ethnicity were not significant in any of the models. Signal strength was positively associated with average and inferior cRNFL measurements $(\mathrm{p}<0.001$ and $\mathrm{p}=0.016$, respectively). Signal strength did not show any significant relationship with other OCT parameters.

VF MD and VFI rate of change were statistically significant, $-0.48 \pm 0.07 \mathrm{~dB} / \mathrm{yr}(\mathrm{p}<0.001)$ and $-1.80 \pm 0.26 \% / \mathrm{yr}(\mathrm{p}<0.001)$, respectively. The OCT cRNFL rates did not reach statistical significance (Table 2). At the same time period, macular and $\mathrm{ONH}$ parameters demonstrated statistically significant rates of change. In the sensitivity analysis, no statistically significant quadratic effect of follow-up length on progression rate was noted in any of the parameters except for average $\mathrm{C} / \mathrm{D}$ ratio, for which subjects with longer followup had faster progression rate $(\mathrm{p}=0.022)$.

Figure 1 depicts a representative subject with advanced glaucoma. The subject's VFI progressed significantly on the trend analysis of the guided progression analysis (GPA) (A). The GPA of the RNFL and ONH (B) did not demonstrate significant rates of progression for the RNFL (average, inferior and superior), but the rate for C/D ratio and changes on the deviation maps were found to be significant. The GPA of the macula (C) also showed significant rates of progression in average, inferior and superior GCIPL.

\section{Discussion}

Our study evaluated the rates of progression of peripapillary, macular and $\mathrm{ONH}$ parameters that are provided by a commercially available SD-OCT in a cohort of subjects with advanced structural glaucomatous damage. The current diagnostic strategies to detect progression in advanced glaucoma present substantial challenges. Considering the high risk of this group to develop debilitating vision and functionality, it is of utmost importance to use sensitive tools for detecting disease progression. We demonstrated that macular and ONH parameters along with VF testing show progression even in eyes with advanced structural glaucomatous damage, even in eyes that reached the floor effect level with the commonly used cRNFL thickness measurement. 
The study was designed to reproduce the clinical situation where the cRNFL is approaching or has reach the "floor effect" with baseline cRNFL $<60 \mu \mathrm{m}$. The baseline mean cRNFL in our cohort was $54.61 \pm 3.48 \mu \mathrm{m}$, slightly below the $57 \mu \mathrm{m}$ threshold of floor effect, as reported by Mwanza et al. ${ }^{16}$ It should be noted that while the structural cRNFL thickness damage was substantial, the median baseline VF MD of our cohort was only $-10.20 \mathrm{~dB}$ due to the well documented structure-function disparity in glaucoma.

We demonstrated a statistically significant rate of progression of GCIPL (average, superior and inferior) despite the "floor effect" of the cRNFL. These results are in agreement with Belghith et al., who demonstrated a significant rate of progression for GCPIL in eyes with very advanced glaucoma defined by VF MD $\leq-21 \mathrm{~dB} .{ }^{17}$ Bowd et al. demonstrated in a population of advanced glaucoma (defined by MD $\leq-12 \mathrm{~dB}$ ) that GCIPL had a significant rate of change of $-0.21 \mu \mathrm{m} / \mathrm{year}$. They also showed that the percentage of area that had not reached the floor at baseline was larger for the GCIPL than cRNFL and the ONH's minimum rim width (MRW) ${ }^{18}$ Shin et al. demonstrated that the GCIPL GPA detected significant differences between the rates of progression for a group of subjects with visual field progression versus those that did not show progression in a moderate to advanced glaucoma sub-group, while the differences of the cRNFL rates were not significant. ${ }^{19}$

The ability of GCIPL to trace glaucoma structural progression during late stage disease when no significant change is detected by cRNFL may reflect that the damage impacting the GCIPL thickness occurs at a different rate and at a later stage of disease in comparison with cRNFL. Notwithstanding, the floor effect of the GCIPL may also be present, thus limiting the utility of this parameter in some patients reaching the floor effect of the cRNFL.

ONH parameters, especially the rim area, demonstrated a significant rate of change in our study. We also observed changes in cup volume and C/D ratio (average and vertical) that were small, yet statistically significant. Several studies demonstrated the ability of OCT's ONH parameters to detect progression, but not specifically in advanced disease. ${ }^{8,20}$ The changes in the $\mathrm{ONH}$ in advanced glaucoma may be indicative that excavation and neural tissue loss in the optic nerve continues even at this late stage. The role of lamina cribrosa and prelaminar tissue analysis in monitoring glaucoma progression requires further investigation. $^{21-24}$

OCT and VF GPA is a clinically useful tool for tracking glaucoma longitudinally. ${ }^{10}$ However, this approach has limitations for subjects with advanced glaucoma. The reduced reproducibility in VF areas of low threshold sensitivity limiting the ability to analyze areas with a deeper loss of sensitivity, which limits the VF usefulness in this subset of subjects. ${ }^{13}$ The OCT GPA for advanced glaucoma also has limitations, given the aforementioned "floor effect" of the cRNFL. Notwithstanding, in our sample, 34 out of 44 eyes demonstrated significant change in the event analysis of the ONH GPA, where no further cRNFL progression was detected with the trend analysis. Thus, evaluating changes in areas outside the circle within the RNFL map might be useful even in the structurally advanced subset of glaucoma. This might be related to spatially varying dynamic range of RNFL thickness measurements or lower specificity of the event analysis. It should also be noted that the Cirrus GPA does not account for the effect of age-related loss of cRNFL, GCIPL and ONH 
parameters. ${ }^{25}$ A previous study demonstrated that age-related loss could explain a large proportion of the deterioration observed in progressing eyes. ${ }^{26}$ However, the impact of agerelated loss in advanced glaucoma with its restricted dynamic range of tissue loss is yet to be determined. In order to prevent possible confounders due to the presence of co-morbidities, we imposed the criterion usually used in this type of study and disqualified subjects with comorbidities. In a cohort with advanced glaucoma, which typically includes a large percentage of aging participants, this criterion might limit the applicability of the results to real life clinical populations. Further studies are required to specifically address the confounding effect of these comorbidities. ${ }^{27}$

In conclusion, macular GCIPL and ONH parameters can be useful for detecting and following glaucoma progression in subjects with structurally advanced glaucoma.

\section{Acknowledgments}

Supporting Grant: NIH R01-EY013178 (Bethesda, MD)

\section{References}

1. Weinreb RN, Aung T, Medeiros FA. The pathophysiology and treatment of glaucoma: a review. JAMA. 2014; 311:1901-11. [PubMed: 24825645]

2. Sung KR, Wollstein G, Kim NR, et al. Macular assessment using optical coherence tomography for glaucoma diagnosis. Br J Ophthalmol. 2012; 96:1452-5. [PubMed: 23018425]

3. Leung CK. Diagnosing glaucoma progression with optical coherence tomography. Curr Opin Ophthalmol. 2014; 25:104-11. [PubMed: 24370973]

4. Dong ZM, Wollstein G, Schuman JS. Clinical Utility of Optical Coherence Tomography in Glaucoma. Invest Ophthalmol Vis Sci. 2016; 57:OCT556-67. [PubMed: 27537415]

5. Grewal DS, Tanna AP. Diagnosis of glaucoma and detection of glaucoma progression using spectral domain optical coherence tomography. Curr Opin Ophthalmol. 2013; 24:150-61. [PubMed: 23328662]

6. Yang Z, Tatham AJ, Zangwill LM, et al. Diagnostic ability of retinal nerve fiber layer imaging by swept-source optical coherence tomography in glaucoma. Am J Ophthalmol. 2015; 159:193-201. [PubMed: 25448991]

7. Bussel II, Wollstein G, Schuman JS. OCT for glaucoma diagnosis, screening and detection of glaucoma progression. Br J Ophthalmol. 2014; 98(Suppl 2):ii15-9. [PubMed: 24357497]

8. Na JH, Sung KR, Lee JR, et al. Detection of glaucomatous progression by spectral-domain optical coherence tomography. Ophthalmology. 2013; 120:1388-95. [PubMed: 23474248]

9. Wollstein G, Schuman JS, Price LL, et al. Optical coherence tomography longitudinal evaluation of retinal nerve fiber layer thickness in glaucoma. Arch Ophthalmol. 2005; 123:464-70. [PubMed: 15824218]

10. Leung CK, Cheung CY, Weinreb RN, et al. Evaluation of retinal nerve fiber layer progression in glaucoma: a study on optical coherence tomography guided progression analysis. Invest Ophthalmol Vis Sci. 2010; 51:217-22. [PubMed: 19684001]

11. de Moraes CG, Liebmann JM, Medeiros FA, Weinreb RN. Management of advanced glaucoma: Characterization and monitoring. Surv Ophthalmol. 2016; 61:597-615. [PubMed: 27018149]

12. Hood DC, Kardon RH. A framework for comparing structural and functional measures of glaucomatous damage. Prog Retin Eye Res. 2007; 26:688-710. [PubMed: 17889587]

13. Gardiner SK, Swanson WH, Goren D, et al. Assessment of the reliability of standard automated perimetry in regions of glaucomatous damage. Ophthalmology. 2014; 121:1359-69. [PubMed: 24629617] 
14. Gardiner SK. Effect of a variability-adjusted algorithm on the efficiency of perimetric testing. Invest Ophthalmol Vis Sci. 2014; 55:2983-92. [PubMed: 24713484]

15. Brusini P, Johnson CA. Staging functional damage in glaucoma: review of different classification methods. Surv Ophthalmol. 2007; 52:156-79. [PubMed: 17355855]

16. Mwanza JC, Kim HY, Budenz DL, et al. Residual and Dynamic Range of Retinal Nerve Fiber Layer Thickness in Glaucoma: Comparison of Three OCT Platforms. Invest Ophthalmol Vis Sci. 2015; 56:6344-51. [PubMed: 26436887]

17. Belghith A, Medeiros FA, Bowd C, et al. Structural Change Can Be Detected in AdvancedGlaucoma Eyes. Invest Ophthalmol Vis Sci. 2016; 57:OCT511-8. [PubMed: 27454660]

18. Bowd C, Zangwill LM, Weinreb RN, et al. Estimating Optical Coherence Tomography Structural Measurement Floors to Improve Detection of Progression in Advanced Glaucoma. Am J Ophthalmol. 2017; 175:37-44. [PubMed: 27914978]

19. Shin JW, Sung KR, Lee GC, et al. Ganglion Cell-Inner Plexiform Layer Change Detected by Optical Coherence Tomography Indicates Progression in Advanced Glaucoma. Ophthalmology. 2017; 124:1466-74. [PubMed: 28549518]

20. Kostanyan T, Sung KR, Schuman JS, et al. Glaucoma Structural and Functional Progression in American and Korean Cohorts. Ophthalmology. 2016; 123:783-8. [PubMed: 26778345]

21. Wu Z, Lin C, Crowther M, et al. Impact of Rates of Change of Lamina Cribrosa and Optic Nerve Head Surface Depths on Visual Field Progression in Glaucoma. Invest Ophthalmol Vis Sci. 2017; 58:1825-33. [PubMed: 28353690]

22. Chung HS, Sung KR, Lee JY, Na JH. Lamina Cribrosa-Related Parameters Assessed by Optical Coherence Tomography for Prediction of Future Glaucoma Progression. Curr Eye Res. 2016; 41:806-13. [PubMed: 26268599]

23. Kim TW, Kagemann L, Girard MJ, et al. Imaging of the lamina cribrosa in glaucoma: perspectives of pathogenesis and clinical applications. Curr Eye Res. 2013; 38:903-9. [PubMed: 23768229]

24. Kim YW, Jeoung JW, Kim YK, Park KH. Clinical Implications of In Vivo Lamina Cribrosa Imaging in Glaucoma. J Glaucoma. 2017; 26:753-61. [PubMed: 28787290]

25. Sung KR, Wollstein G, Bilonick RA, et al. Effects of age on optical coherence tomography measurements of healthy retinal nerve fiber layer, macula, and optic nerve head. Ophthalmology. 2009; 116:1119-24. [PubMed: 19376593]

26. Vianna JR, Danthurebandara VM, Sharpe GP, et al. Importance of Normal Aging in Estimating the Rate of Glaucomatous Neuroretinal Rim and Retinal Nerve Fiber Layer Loss. Ophthalmology. 2015; 122(12):2392-8. [PubMed: 26421707]

27. Chhablani J, Sharma A, Goud A, et al. Neurodegeneration in Type 2 Diabetes: Evidence From Spectral-Domain Optical Coherence Tomography. Invest Ophthalmol Vis Sci. 2015; 56:6333-8. [PubMed: 26436886] 
A
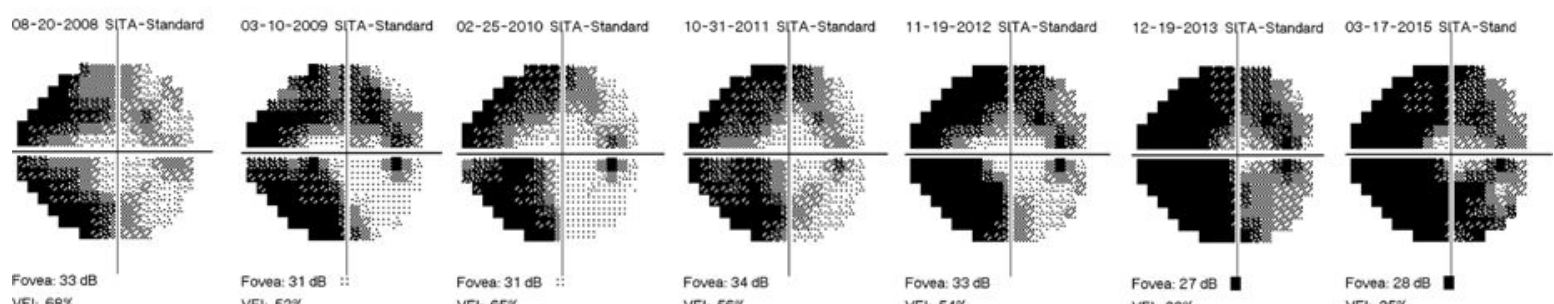

VFI: $68 x$

VFI: $56 \%$

VFI: $54 \%$

Fovea: 27

VFI: $25 \%$

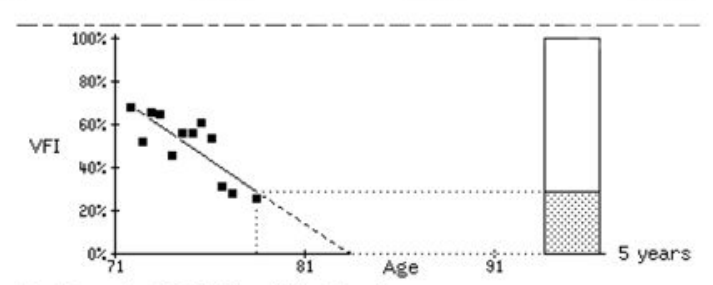

Rato of Progression $-5.9 \pm 3.1 \% /$ year ( $95 \%$ confidence)

Slope significant at $P<1 \%$

B

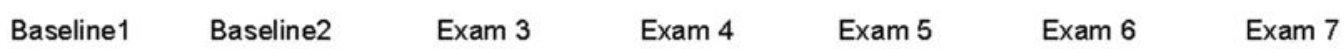
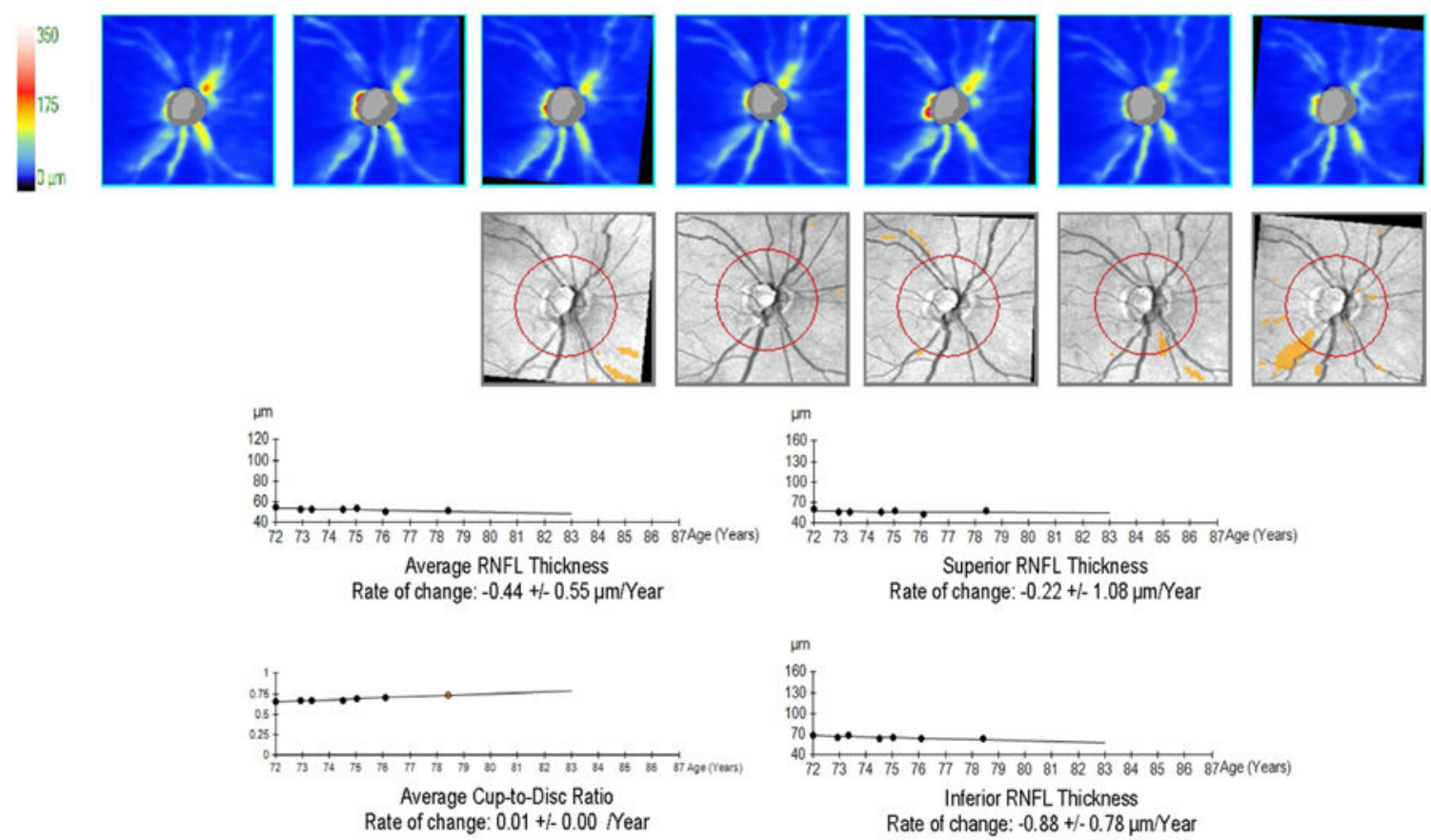

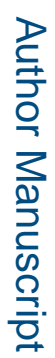
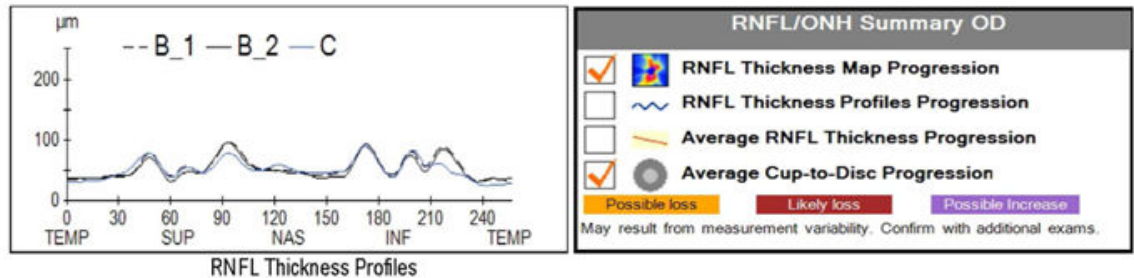

Ophthalmology. Author manuscript; available in PMC 2019 December 01. 


\section{C}
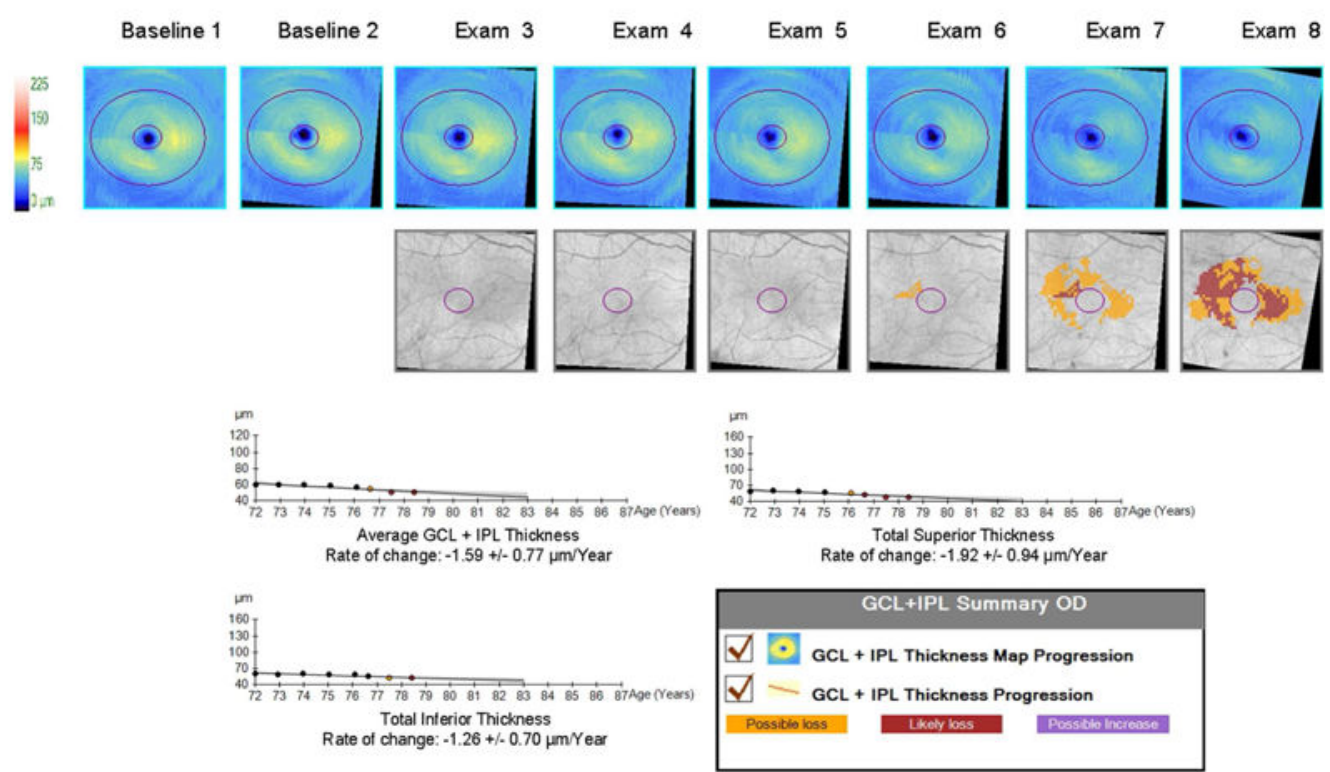

Figure 1.

Demonstrative subject with advanced glaucoma showing a significant rate of progression for VFI (A); Guided progression analysis (GPA) showing no progression with cRNFL but C/D ratio progression in the last two visits and "possible loss" in the RNFL thickness map progression (B); Significant thinning of the macular GCIPL (C). 


\section{Table 1}

Baseline visual field and OCT measurements of the study population.

\begin{tabular}{|l|c|}
\hline Parameter & Mean $($ SD) \\
\hline MD $(\mathrm{dB}){ }^{*}$ & $-10.18(-14.21,-5.51)$ \\
\hline VFI $(\%) *$ & $77.00(62.75,86.00)$ \\
\hline Average cRNFL $(\mu \mathrm{m})$ & $54.55(3.42)$ \\
\hline Superior cRNFL $(\mu \mathrm{m})$ & $62.66(8.55)$ \\
\hline Inferior cRNFL $(\mu \mathrm{m})$ & $57.52(7.66)$ \\
\hline GCIPL $(\mu \mathrm{m})$ & $57.55(6.88)$ \\
\hline Superior GCIPL $(\mu \mathrm{m})$ & $58.14(8.06)$ \\
\hline Inferior GCIPL $(\mu \mathrm{m})$ & $56.43(6.92)$ \\
\hline Rim area $\left(\mathrm{mm}^{2}\right)$ & $0.65(0.17)$ \\
\hline Cup volume $\left(\mathrm{mm}{ }^{3}\right)$ & $0.50(0.35)$ \\
\hline Average CD ratio & $0.78(0.09)$ \\
\hline Vertical CD ratio & $0.80(0.09)$ \\
\hline
\end{tabular}

$\mathrm{MD}=$ mean deviation; $\mathrm{VFI}=$ visual field index; $\mathrm{cRNFL}=$ circumpapillary retinal nerve fiber layer; $\mathrm{GCIPL}=$ ganglion cell inner plexiform layer; $\mathrm{CD}$ ratio=cup-to-disc ratio.

Median $(25 \%, 75 \%$ quartiles). 


\section{Table 2}

OCT parameters rate of change

\begin{tabular}{|l|c|c|}
\hline Parameter & Rate of change per year $(\mathrm{SE})$ & $\mathbf{p}$ \\
\hline Average cRNFL $(\mu \mathrm{m})$ & $0.009(0.064)$ & 0.885 \\
\hline Superior cRNFL $(\mu \mathrm{m})$ & $0.053(0.129)$ & 0.682 \\
\hline Inferior cRNFL $(\mu \mathrm{m})$ & $-0.197(0.121)$ & 0.106 \\
\hline GCIPL $(\mu \mathrm{m})$ & $-0.573(0.051)$ & $<0.001$ \\
\hline Superior GCIPL $(\mu \mathrm{m})$ & $-0.702(0.051)$ & $<0.001$ \\
\hline Inferior GCIPL $(\mu \mathrm{m})$ & $-0.463(0.052)$ & $<0.001$ \\
\hline Rim area $\left(\mathrm{mm}^{2}\right)$ & $-0.010(0.001)$ & $<0.001$ \\
\hline Cup volume $\left(\mathrm{mm}^{2}\right)$ & $0.008(0.001)$ & $<0.001$ \\
\hline Average CD ratio ${ }^{*}$ & $0.006(0.001)$ & $<0.001$ \\
\hline Vertical CD ratio & $0.006(0.001)$ & $<0.001$ \\
\hline
\end{tabular}

$\mathrm{MD}=$ mean deviation, $\mathrm{VFI}=\mathrm{visual}$ field index, $\mathrm{cRNFL}=$ circumpapillary retinal nerve fiber layer, $\mathrm{GCIPL}=$ ganglion cell inner plexiform layer, $\mathrm{CD}$ ratio=cup-to-disc ratio.

Significant quadratic term of follow-up length (0.022). 